Fourth International Conference on Sustainable Construction Materials and Technologies

http://www.claisse.info/Proceedings.htm

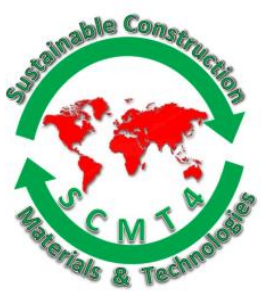

SCMT4

Las Vegas, USA, August 7-11, 2016

\title{
The Plate Test carried out on Fresh Self- Compacting Concrete
}

\author{
Sofiane Amziane ${ }^{1}$, Arnaud Perrot ${ }^{2}$ \\ ${ }^{1}$ LIMat B, Université de Bretagne Sud, Lorient France, Email: sofiane.amziane@univ-bpclermont.fr, \\ ${ }^{2}$ Clermont Université, Institut Pascal, Clermont-Ferrand, France, Email: 〈arnaud.perrot@univ-ubs.fr〉.
}

\begin{abstract}
To monitor, these entire physical phenomenon linked to cement setting, several tools are needed from rheometer, transducer, calorimeter and others expensive and sometimes very difficult set up. The plate test can be a simple and cheap substitute for all these tools and can provide interesting information about structural build-up and setting. This device consists of a steel rough plate immersed in the cement-based materials and was initially developed to enable a simpler measurement of the yield stress increase during the dormant period. After providing and explaining the test physical background and procedure, we here try to extend the test duration to get information on both the thermal dilatation and the setting time. Due to the deformation of the material at rest and due to changes in the volume linked to thermal expansion, the plate apparent mass varies with time. The apparent shear stress value and the thermal stress of the cement paste could be deduced from this measurement.
\end{abstract}

\section{INTRODUCTION}

Despite the long tradition of characterizing cement paste time evolution by the initial and final setting time, these values are not sufficient to answer some of the more practical questions related with constructability. The answer to these questions is of paramount importance for the civil engineer to design a concrete meeting a given construction schedule. However, to answer the above questions, the flow properties of the material need to be known. In contrast with a mechanical method that will be always dependent on the geometry and the applied forces, the proposed investigation was carried out on the idea that it was possible to monitor the setting through the variation of yield stress. Roussel and other authors have shown that during the dormant period and at rest, SCC structure build-up and its yield stress increases (Roussel 2006), (Roussel 2005), (Roussel 2012), (Wallevik 2009). This increase is responsible for lot of practical application such as multilayer casting, 3D printing, formwork pressure decay... (Roussel 2006), (Billberg 2014), (Amziane 2008). The increase rate of yield stress is commonly determined using rheological measurements carried out after different resting time. Portable system has been developed to monitor the yield stress increase on site (Khayat 2012). Another strategy is to use the plate test measurement (Billberg 2014). The plate test device, consisting of a rough plate (or needle) immersed in the fluid sample, was developed to enable a simpler measurement of the yield stress increase of non-Newtonian suspensions such as self-compacting concrete. Due to the deformation of the material at rest along the vertical axis, shearing appears at the plate surface inducing the plate apparent mass to vary with time. 
The plate test device has been first used for concrete in 2008 (Amziane 2008), (Tchamba 2008). The problem is the same as the steel rebar immersion in concrete formwork (Perrot 2009). The elastoplastic properties and deformation of the fresh cement paste are used. Due to local vertical deformation of the cement paste at very early age (shrinkage, dilatation, settlement), stresses are mobilized at the interface between the paste and the rough plate. This induces variation of the apparent mass of the plate or needle, as proposed by Sleiman et al. (Sleiman 2010). Moreover, since the plate or needle is static and the material shrinkage is slow, the dynamic effects are, therefore, negligible. The plate tests measurements has also been shown to be able to give accurate values of the setting times (Sleiman 2010). The paper aims to provide a correct protocol for performing a plate test measurement carried out on SCC that is able to provide the rate of increase of yield stress during the dormant period, the setting times. Moreover, we show that the plate test is able to detect the thermal dilatation due to exothermic hydration of the cement particles. We also try to give some limitations of this test that is able to provide important data when properly carried out.

\section{Experimental program}

The plate test device, a multiform apparatus. The device is composed of a needle or a plate (solid body) rigidly attached below a fixed support. The (solid body) is lowered into a frustum, containing the material. The apparent mass of the needle or the apparent mass of the sample is continuously monitored versus time by recording the balance output with a computer. The immersed tool used can be a needle with a diameter of $1.13 \mathrm{~mm} \pm 0.05 \mathrm{~mm}$ and $40 \mathrm{~mm}$ long (i.e. a roughened Vicat needle) (Sleiman 2010), can be a plate covered by sandpaper (Tchamba 2008) (Amziane 2008) or also a 10mm diameter rebar (Billberg 2014) (Sleiman 2010). The distance between the needle/plate and the frustum walls is large enough that there is no influence on the stress measured due to the size of the frustum as shown by (Zhang 2010) and Tchamba et al. (Tchamba 2008). Moreover, in order to ensure that the yield stress is fully mobilized at the interface with the immersed tool, it is critical to make sure that the critical strain is overcome at the interface. This can be made by having a sufficient length of concrete under the immersed tool. Assuming a linear settlement of concrete with sample height, figure 1 obviously describes the increase of the strain at the interface with the sample height.

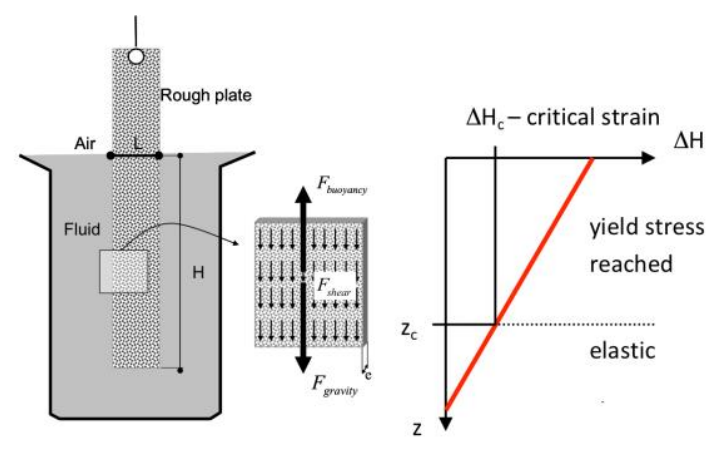

Figure 1. Evolution of the settlement $\Delta H$ within the sample. The yield stress is fully mobilized only over a critical depth zc corresponding to a critical settlement $\Delta H \mathbf{H}$ for which the concrete critical strain is obtained.

It is important to note that the balance can be used to measure the immersed tool apparent mass and the sample apparent mass as shown on figure 3 for two possible test configurations. In this work, we have performed two series of tests using two different apparatus. For all tests, a thermocouple was introduced into the self-compacting materials in order to measure the thermal activity of the material during the whole test. 
The first configuration consists of the static Vicat needle device used in Sleiman et al. work. The second configuration is designed to be dedicated to mortar and concrete. The measurement sequence begins with the immersion of the plate in a suspension filled vessel. Then length of the immersed portion of the tool is measured before the start of the test.

Measurement precision and reproducibility depend on the following parameters: a) immersion depth (precision: $1 \mathrm{~mm}$ ), b) measured mass (precision: $0.1 \mathrm{~g}$ ) and c) experimental conditions such as temperature and relative humidity. Variations between tests performed on the same material in the same experimental conditions are less than $5 \%$. The immersed tool surface is lightly roughened or covered with sandpaper to avoid slippage effects. In addition for the Vicat static test, we measure the material deformation with a comparator placed on the frustum material. We used a small steel grill placed between the comparator and the cement paste to reduce the error due to comparator weight. We followed the evolution of deformation with time to check if the settlement is sufficient to reach the critical strain at the interface. A linear deflectometer, LVDT, attached by fixed support, is placed on the top surface of this frustum. This LVDT is used to monitor surface settlement. Tests are carried out during 24 hours.

Materials. The first serie of tests is performed on normal consistency cement pastes. The cement (CEM $\mathrm{I} / 52.5 \mathrm{~N}$ ) used contains mass fractions of $95 \%$ clinker, $3.5 \%$ gypsum and $1 \%$ filler. The specific Blaine surface is $425 \mathrm{~m}^{2} / \mathrm{kg}$. The cement was prepared in a $5 \mathrm{~L}$ mixer according to the standard ISO 9597 . The second serie is performed on a self-compacting concrete. For this concrete, A CEM I $42.5 \mathrm{~N}$ is used at a recipe of $420 \mathrm{~kg} / \mathrm{m}^{3}$. A limestone filler is added $\left(120 \mathrm{~kg} / \mathrm{m}^{3}\right)$ and the naturally rounded aggregate is of granitic origin (sand + coarse aggregate $1700 \mathrm{~kg} / \mathrm{m}^{3}$ ). A poly carboxylate ether (PCE) plasticizer was used in this study. The W/C ratio is 0.385 . All computed yield stress from plate tests are compared with a reference yield stress measured on a BOHLIN Gemini®200 viscometer equipped with a Vane geometry following the procedure described in (Perrot 2012) with an apparent shear rate of $0.001 \mathrm{~s}^{-1}$.

Data analysis - general equation. The data analysis is based on the force balance equation of a static cylinder (Figure 1). Three phenomena are acting on the plate: gravity, buoyancy and shearing at the material/plate interface. In air, the mass plate $\mathrm{M}_{0}$ is only due to gravity (1) and does not change with time:

$$
M_{0}(t) \times \vec{g}=\overrightarrow{F_{\text {gravity }}}
$$

For an immersed plate, the mass measured corresponds to the apparent mass $M(t)$, which can deduced from the static equilibrium (2) of the tool in a yield stress fluid:

$$
M(t) \times \vec{g}=\overrightarrow{F_{\text {gravity }}}+\overrightarrow{F_{\text {buoyancy }}}+\overrightarrow{F_{\text {shear }}}-\alpha \cdot G \cdot \Delta T \cdot \vec{z}
$$

where $\mathrm{F}_{\text {gravity }}$ is the constant weight of the plate, $\mathrm{F}_{\text {buoyancy }}$ is the resistance force due to buoyancy and $\mathrm{F}_{\text {shear }}$ is the resistance due to shearing at the material/cylinder interface, $\alpha . G . \Delta T . \vec{z}$ is a correction term taking into account the thermal dilatation which might not be negligible during the hydration period. $G$ is an apparent shear modulus and $\alpha$ is the dilatation coefficient depending only hydration time. In the present case (i.e. A Vicat needle), it is important to note that the bottom end effect is neglected because the cylinder is very thick (needle) or empty (cylinder). This assumption is not valid for higher diameter value (Boujlel 2012) (Lootens 2009), (Perrot 2011). In this case, we advise to slightly move the plate upward in order to create an empty space between the bottom end of the immersed tool and the concrete. 
It is important to note here that, as opposed to a penetrometer test (Vicat needle), the plate is perfectly static. This test is not really intrusive because the only movement is due to the changes occurring in the material. In other words the plate behaves as an additional vessels wall. In the general case, the particles in a suspension move downwards creating a higher density layer at the bottom. Equation 2 can be rewritten as follow (3) if considerations are taken that the force applied on the cylinder change with time due to sedimentation:

$$
M(t, T)=\frac{1}{g}\left(F_{\text {gravity }}(t)-F_{\text {buoyancy }}(t)+F_{\text {shear }}(t)-F_{\text {dilation }}(t, T)\right)
$$

As a result, the mass variation (4) induced by the plate immersion is:

$$
\Delta M(t, T)=\frac{1}{g}\left(-F_{\text {buoyancy }}(t)+F_{\text {shear }}(t)-F_{\text {dilation }}(t, T)\right)
$$

If the material remains homogeneous, the buoyancy remains constant during time.

\section{Raw data}

Consolidation diagram. The consolidation of the cement paste under its own weight is possible as long as the material has not yet stiffened and is recorded with the LVDT. Thixotropy and then structural build up gradually reduces the rate of consolidation as shown by Sleiman et al. (Sleiman 2010) The stress recorded by the needle corresponds to the yield stress as soon as the paste settlement is more than 0.005 (just after the test began). This means that before this time, the yield stress is not mobilized wherever on the needle surface. In order to reduce and avoid this duration, we advise, another time, to slightly move the immersed needle upward. For all performed tests, the time needed to overcome the critical strain is always lower than 15 minutes.

Mass/Temperature-Time diagram. Three stages can be identified on the diagram mass vs. time (Figure 2) recorded with the static Vicat needle device. The first stage corresponds to a low thermal activity. The mass variation is attributed to the entire phenomena occurring during the setting period (thixotropy, consolidation and plastic shrinkage). Around 5 hours, the thermal activation begins inducing the swelling of the cement paste. The "stage two" can be identified as the swelling period with an inversion of the stress at the plate. After that, stage 3 shows that the temperature goes down and the mass and the temperature tend toward a stabilized value. Moreover, we can observe that the temperature peak is simultaneous with the recorded minimum mass variation. Then, it shows that both local extrema are linked .Now that we have examined the raw data, we can go a step further and see what information we can obtained from the plate tests measurement.

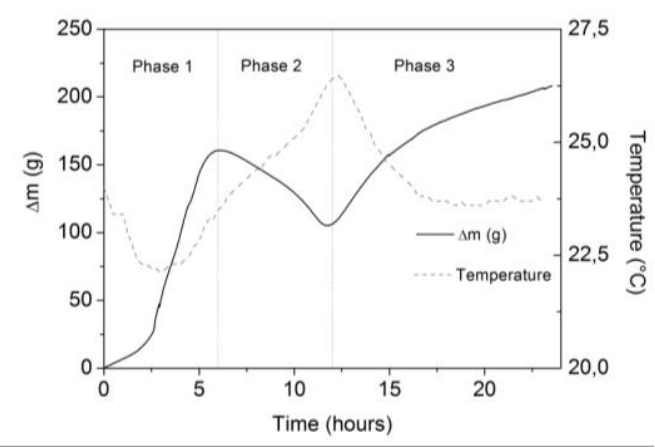

Figure 2. Mass-Temperature vs.Time diagrams recorded using the static Vicat device / different stages of behavior. 


\section{Discussion and analysis of the raw data}

\section{Rheology and structural buildModelling of the thermal expansion}

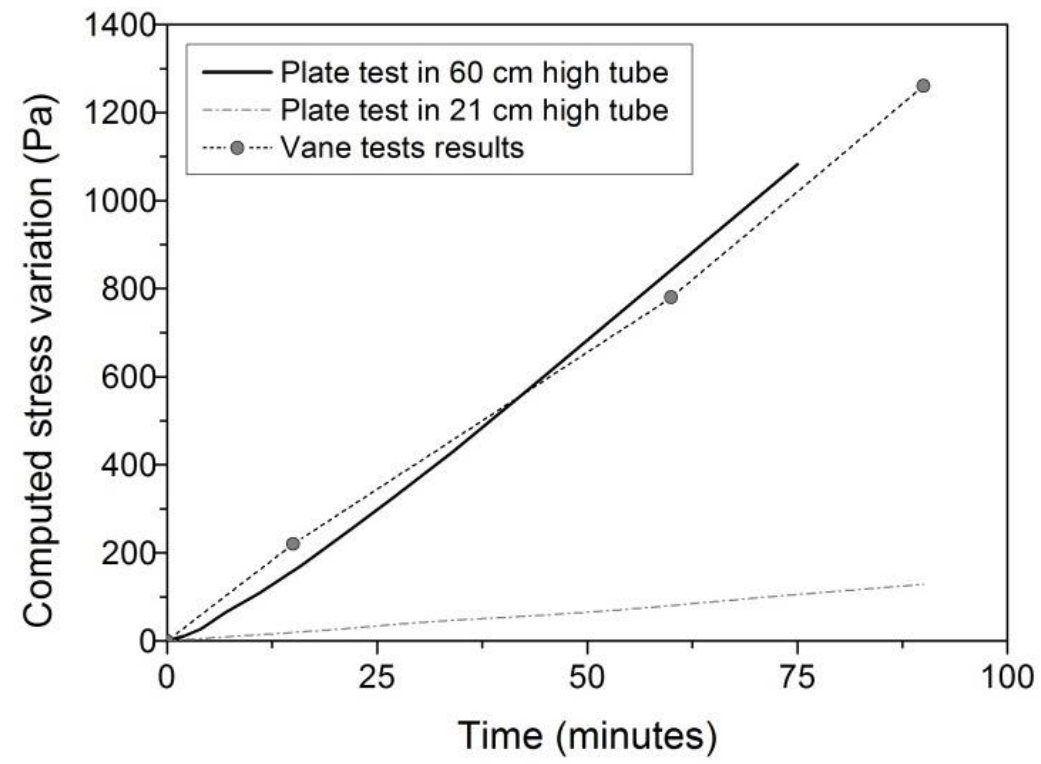

Figure 3. Stress variation computed from data of tests performed with concrete. Tests performed with two different sample heights $(21$ and $60 \mathrm{~cm})$. Results compared with rheological vane tests measurement.

During the thermal expansion stage, the critical strain is no longer reached due to the paste swelling. Then, in the second stage, the computed modulus does not correspond to the intrinsic shear modulus but to an apparent shear modulus that depends on the test geometry (5). In this stage, the material is not able to flow on its own weight and part of the immersed tool interface is irreversibly broken.

$$
G_{\text {apparen }}(t)=\frac{g \Delta M(t)+\rho g V}{\varepsilon_{c} \cdot S-\alpha \Delta t}
$$

Then, equation (5) is used to fit a value of that erases the effect of thermal dilation on the evolution on the apparent shear modulus. A value of 0.005 is found and is in agreement with values found in the literature (Loukili 2000). Then, the evolution of the shear modulus can be obtained during the first 24 hours (Figure 4). We can see on figure 4 that the effect of thermal expansion has to be taken into account in order to accurately describe the paste hardening. A shear modulus computed with equations $(4,5)$ that does not take into account the thermal expansion is also computed. Then, the difference obtained between the shear moduli computed with and without taking into account the thermal expansion at 24 hours is due to the difference of the paste temperature at this time. It is worth noting that the difference between both shear modulus obtained with both computation follow the temperature evolution of the paste given in figure 2 . 


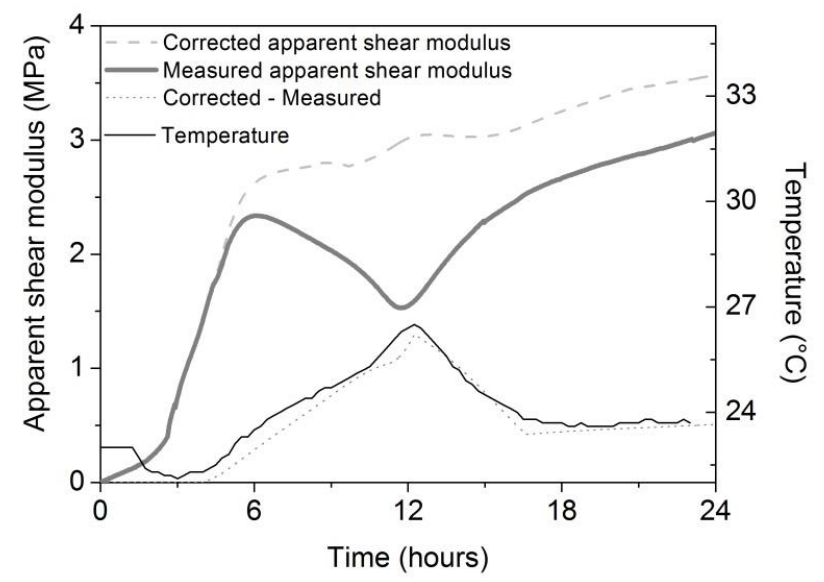

Figure 4. Evolution of the apparent shear moduli. The grey line corresponds to the measured one. The dashed line corresponds to a corrected apparent shear moduli that takes into account thermal expansion.

\section{CONCLUSION}

The main scope of the paper was to examine the possibility of using alternative tests to the classical methods to monitor early age behaviour of SCC from casting to hardening. It was shown that plate test method tested on SCC is very promising as they correlate well with rheological test and thermal variation measurement, providing more information with a unique test.

\section{REFERENCES}

Amziane, S. Perrot, A. and Lecompte, T. (2008) A novel settling and structural build-up measurement method, Measurement Science and Technology, Vol. 19, p. 105702.

Billberg, P.H. Roussel, N. Amziane, S. Et al. (2014) Field validation of models for predicting lateral form pressure exerted by SCC, Cement and Concrete Composites, Vol 54, p. 70-79.

Boujlel, J. and Coussot, P. (2012) Measuring yield stress: a new, practical, and precise technique derived from detailed penetrometry analysis, Rheologica Acta, Vol. 51, p. 867-882.

Khayat, K.H. Omran, A.F.. Naji, S. Billberg, P. and Yahia, A. (2012) Field-oriented test methods to evaluate structural build-up at rest of flowable mortar and concrete, Materials and Structures, Vol. 45, p. 15471564.

Lootens, D. Jousset, P. Martinie, L. et al. (2009) Yield stress during setting of cement pastes from penetration tests, Cement and Concrete Research, Vol. 39, p. $401-408$.

Loukili, A. Chopin, D. Khelidj, A. and Le Touzo J.Y. (2000) A new approach to determine autogenous shrinkage of mortar at an early age considering temperature history, Cement and Concrete Research, Vol. 30, p. 915-922.

Perrot, A. Amziane, S. Ovarlez, G. and Roussel, N. (2009) SCC formwork pressure: Influence of steel rebars, Cement and Concrete Research, Vol. 39, p. $524-528$. 
Perrot, A. Mélinge, Y. Estellé, P. et al. (2011) The back extrusion test as a technique for determining the rheological and tribological behaviour of yield stress fluids at low shear rates, Applied Rheology, Vol. 21, p. 53642.

Perrot, A. Lecompte, T. Khelifi, H. et al. (2012) Yield stress and bleeding of fresh cement pastes, Cement and Concrete Research, Vol. 42, p. 937 - 944.

Perrot, A. Pierre, A. Vitaloni, S. and Picandet, V. (2015) Prediction of lateral form pressure exerted by concrete at low casting rates, Materials and Structures, Vol. 48, p. 2315-2322.

Perrot, A. Rangeard, D. and Pierre A. (2015) Structural built-up of cement-based materials used for 3Dprinting extrusion techniques, Materials and Structures.Available online.

Roussel, N. (2005) Steady and transient flow behaviour of fresh cement pastes, Cement and Concrete Research, Vol. 35, p. 1656 - 1664.

Roussel, N. (2006) A thixotropy model for fresh fluid concretes: Theory, validation and applications, Cement and Concrete Research, Vol. 36, p. 1797 - 1806.

Roussel N. and Cussigh F. (2008) Distinct-layer casting of SCC: The mechanical consequences of thixotropy, Cement and Concrete Research, Vol. 38, p. 624-632.

Roussel, N. Lemaitre, A.. Flatt, R.J. and Coussot, P. (2010) Steady state flow of cement suspensions: A micromechanical state of the art, Cement and Concrete Research, Vol. 40, p. 77-84.

Roussel, N. Ovarlez, G. Garrault, S. and Brumaud, C. (2012) The origins of thixotropy of fresh cement pastes, Cement and Concrete Research. Vol. 42, p. 148 - 157.

Sleiman, H. Perrot,A. and Amziane, S. (2010) A new look at the measurement of cementitious paste setting by Vicat test, Cement and Concrete Research. Vol. 40, p. $681-686$.

Tchamba, J.C. Amziane, S. Ovarlez, G. and Roussel, N. (2008) Lateral stress exerted by fresh cement paste on formwork: Laboratory experiments, Cement and Concrete Research, Vol. 38, p. 459 - 466.

Wallevik, J.E. (2009) Rheological properties of cement paste: Thixotropic behavior and structural breakdown, Cement and Concrete Research, Vol. 39, p. 14 - 29.

Zhang, M.H. Ferraris, C. Zhu, H. Et al. (2010) Measurement of yield stress for concentrated suspensions using a plate device, Materials and Structures, Vol. 43, p. 47-62. 